\title{
Design of aerial fog computing with fixed-wing UAVs
}

This paper was downloaded from TechRxiv (https://www.techrxiv.org).

\section{LICENSE}

CC BY-NC-SA 4.0

SUBMISSION DATE / POSTED DATE

26-02-2022 / 03-03-2022

\section{CITATION}

Cardoso da Silva, Rodrigo Augusto; da Fonseca, Nelson Luis Saldanha (2022): Design of aerial fog computing with fixed-wing UAVs. TechRxiv. Preprint. https://doi.org/10.36227/techrxiv.19241850.v1

$\mathrm{DOI}$ 


\title{
This work has been submitted to the IEEE for possible publication. Copyright may be transferred without notice, after which this version may no longer be accessible.
}

\section{Design of aerial fog computing with fixed-wing UAVs}

\author{
Rodrigo A. C. da Silva and Nelson L. S. da Fonseca
}

\begin{abstract}
One challenge in the deployment of a fog computing infrastructure is the support of demands which are variable in time and space as this can temporarily overload fog nodes. Mobile fog nodes can be promptly dispatched to support dynamic demands, helping to cope with overloaded terrestrial infrastructure. Specifically, unmanned aerial vehicles (UAVs) can be used as mobile fog nodes since they benefit from autonomous operation and maneuverability. However, UAVs are typically battery-constrained and can operate only for a limited time. This paper investigates how fixed-wing UAVs can fulfill the role of fog nodes. These UAVs have different aerodynamics and consume less energy than do rotary-wing UAVs. This paper proposes the Spatio-Temporal UAV Fog Node Location algorithm to determine the position of fog nodes mounted on UAVs.
\end{abstract}

\section{Index Terms}

Fog computing, unmanned aerial vehicle, facility location.

\section{INTRODUCTION}

Fog computing relies on fog nodes, which are facilities that host processing, networking, and storage resources on the network edge. One issue in the design of the fog computing infrastructure is the spatial distribution of the resources to serve mobile users since they generate variable demands in terms of time and space. Fog nodes serving locations with high user density may require powerful resources to deal with peaks of user requests, although the resources may remain underloaded most of the time. One way of coping with load variability while maintaining efficient resource utilization is combining fixed and mobile fog nodes [1]. The fixed nodes are constantly connected to energy supplies and offer easy management, while the mobile nodes complement the fixed node resources by moving either to overloaded locations or those with no fog node.

Unmanned aerial vehicles (UAVs) are highly suitable for hosting fog nodes since they can be equipped with both processing and networking resources; this makes it possible to make fog computing capability available to end users on demand [1]-[3]. Moreover, UAVs can be operated remotely and even autonomously. However, UAVs typically have a low autonomy, which limits flight duration to only a couple of minutes before the onboard battery is depleted [4]. Therefore, solutions to extend the operational time of UAVs are necessary. One possible solution is the employment of UAVs connected to the ground by a tether, providing an aircraft with an unlimited energy supply and connectivity to the Internet [5]. Another alternative is by the positioning of UAVs when and where they are needed to cope with such peak demands.

Previous works have proposed the employment of fog nodes deployed on rotary-wing UAVs [1], [6], [7], which can be maintained steady in the air and quickly land on limited spaces. However, their energy consumption is high, which limits the operational time. Alternatively, fixed-wing UAVs can be employed; providing longer operational periods. This extended operational time is essential for fog nodes mounted on UAVs to deal with variable loads. However, fixed-wing UAVs cannot hover; so their trajectory needs to be carefully planned.

This paper aims at evaluating the employment of fixed-wing UAVs as fog nodes, specifically answering the following question: how should fixed-wing UAVs be positioned to provide fog computing infrastructure that can efficiently deal with demands that are variable in time and space as well as maximize the number of processed requests? In an attempt to answer this question, this paper introduces the Spatio-Temporal UAV Fog Node Location (STUFog) algorithm which analyzes the variability of requests and attempts to locate UAVs at locations with high demands, thus maximizing the number of requests processed, minimizing costs, and reducing latency.

The proposal was evaluated carrying out experiments using actual data with variable user demands. Realistic energy consumption and trajectory models for fixed-wing UAVs were considered to determine the location of the fog nodes. Different trajectories were considered to evaluate the effect of the energy consumption and wireless channel on the number of required UAVs. A comparison between deployments with fixed-wing and rotary-wing UAVs is also presented, as well as a discussion of which scenarios benefit most from each type of UAV. The results show that fixed-wing UAVs performing short circular trajectories can be highly efficient in supporting users demands. Moreover, the paper discusses the trade-off between the 
acceptance of requests and network coverage. Tethered deployments are also evaluated as alternatives to reduce the number of UAVs needed.

\section{FIXED-WING AND ROTARY-WING UAV TECHNOLOGIES}

Fixed-wing UAVs are similar to commercial airplanes: the engine generates thrust to move the aircraft forward, and the wings are shaped to generate lift. Fixed-wing UAVs are efficient for longer trajectories since, once in the air, they do not need high power to fly. However, they usually require a runway to take off and land, requiring more complex ground infrastructure. On the other hand, rotary-wing UAVs are vertical take-off and landing vehicles that generate thrust and lift by rotating rotor blades. Both single-rotor or multi-rotor versions are available. Single-rotor UAVs can endure long trips but have less stability to hover. Multi-rotor UAVs are more suitable for hovering and have better maneuverability. However, the greater the number of blades required, the greater is the energy consumption. Most of the existing research on UAVs in wireless networks has considered multi-rotor UAVs.

UAVs flying at low altitudes are typically powered by batteries and have limited autonomy [4]. The operation with lowest energy consumption for fixed-wing UAVs [8] is a straight flight maintaining the same height at a constant speed. Lower speeds lead to lower energy consumption, but fixed-wing UAV flights require a certain minimum speed to generate lift. If the UAV needs to cover a small area, a circular trajectory can be adopted. The larger the radius, the lower is the energy consumption. Rotary-wing UAVs tend to have large energy consumption at high speeds [9], but, if they hover, they significantly reduce such consumption. However, the energy consumed to keep a rotary-wing UAV hovering is usually greater than that to maintain a fixed-wing one flying in a circular trajectory with a large radius.

One solution to overcome the autonomy limitations of UAVs is the employment of tethered UAVs [5]. In such deployments, the UAVs are connected to ground stations (GSs) by a tether that provides both energy supply and connectivity. GSs can be installed either on the ground or on rooftops. However, the tether imposes significant limitations. It limits the distance between the UAV and the GS to the length of the tether, considerably reducing mobility. Moreover, more human intervention is required. Nonetheless, tethered UAVs can be quite advantageous if UAVs are to stay in the same spot for long periods.

Both types of UAVs can carry onboard networking, processing, and storage devices. The network between UAVs and devices on the ground is largely composed of line-of-sight (LoS) links due to the altitude at which the UAVs fly [3], [8], [9], however, longer links lead to greater path loss [10]. If a UAV needs to provide a limited area with wireless coverage or processing, a rotary-wing UAV can be more efficient, since it can hover and maintain a stable wireless channel. Fixed-wing UAVs, however, will have a variable wireless channel. If a user on the ground sends a request to the UAV, they may need to wait for the fixed-wing UAV to come closer, even though the request may be blocked if the delay requirements are not met. Fixed-wing UAVs can also be affected by the Doppler shift, although this phenomenon can usually be compensated in flights at low altitudes and speeds [8].

Fog nodes can be mounted on both fixed-wing and rotary-wing UAVs. Fixed-wing UAVs are usually more energy-efficient than rotary-wing ones, but the latter can provide wireless links with a higher quality of transmission. Rotary-wing UAVs have been broadly considered as part of infrastructure in previous work [1], [6], [7], [9]. Nonetheless, with adequate planning, fixed-wing UAVs can efficiently serve as fog nodes. Fog providers can benefit from their low energy consumption to keep them operational longer, serving more users.

\section{UAVS ON THE NETWORK EDGE}

This section exemplifies the use of UAVs at the network edge rather than being an exhaustive survey on the topic. The authors of [3] have proposed a scheme that uses dynamic power management to reduce the number of active central processing units (CPUs). The solution was based on reinforcement learning; it optimized an objective function that jointly considers energy consumption, job loss probability, and latency.

The design of UAV trajectories is a typical optimization problem [8], [9]. The work in [8] optimized the trajectory of fixed-wing UAVs which need to communicate with ground users. The work in [9], on the other hand, optimized the trajectory of rotary-wing UAVs considering solutions in which communication takes place with both moving and hovering UAVs. The solutions [8] and [9] did not consider, however, UAVs as processing entities.

Other papers [1], [6], [7] have proposed the optimization of the operation of UAVs as processing nodes. Wang et al. [7] proposed an algorithm to decide on the hovering locations for UAVs to provide alternative to fog nodes based on the ground. The authors of [6] proposed an algorithm to select the UAV trajectory as well as task schedule and resource allocation. Finally, in our previous work [1], the extent to which fog nodes on the ground can be replaced by rotary-wing UAVs was explored and a solution to the fog node location problem was proposed which considers both fixed and UAV nodes. The work in [1] did not, however, consider the variable quality of transmission between the UAVs and ground nodes. Although these solutions studied different aspects of aerial fog computing [1], [6], [7], they did not consider the employment of fixed-wing UAVs.

Table I compares the papers reviewed in this section. Numerous studies [1]-[3], [6]-[9] have demonstrated that the deployment of fog nodes on UAVs is feasible but that it must be optimized to prevent battery drain as well as guarantee quality of service for the end users. Nevertheless, most approaches have relied on rotary-wing UAVs, which require high energy consumption 
TABLE I

COMPARISON OF RELATED PAPERS.

\begin{tabular}{l|llll} 
Reference & Research Problem & UAV Type & UAV role & Solution \\
\hline$[3]$ & Active CPUs optimization & Rotary-wing & Edge server & Reinforcement learning \\
{$[8]$} & Trajectory optimization & Fixed-wing & Data collection & Sequential Optimization \\
{$[9]$} & Trajectory optimization & Rotary-wing & Data collection & Successive Convex Approximation \\
{$[7]$} & UAV dispatch & Rotary-wing & Edge server & Heuristic algorithm \\
{$[6]$} & Trajectory and resource allocation & Rotary-wing & Edge server & Successive Convex Approximation and Block Coordinate Descent \\
{$[1]$} & Fog node location & Rotary-wing & Edge server & Mixed-integer linear programming and heuristic \\
\hline The present paper & Fog node location & Fixed-wing & Edge server & Integer linear programming and heuristic
\end{tabular}

to hover or fly from one area to another, which severely reduces operational time. The present paper considers the aspects inherent to fixed-wing UAVs in the proposal of a novel solution to the fog node location problem.

\section{Design OF AERIAL FOG COMPUTING}

This section presents an overview of fog computing based on fixed-wing UAVs. It describes the system model, the fog node location problem, and the solution proposed for the fog node location problem.

\section{A. System model}

Fog nodes usually process end-user requests, reducing the service latency by avoiding the long propagation delays resulting from the need to access cloud data centers. UAVs can be of help in the support of services where terrestrial fog nodes are overloaded, or where fixed node deployment is either impossible or prohibitively expensive.

In this paper, fixed-wing UAVs are used to process requests of users at different locations. Fixed-wing UAVs cannot hover or land in limited spaces; thus, in this paper, they are assumed to perform a circular trajectory with a predefined radius centered on a specific geographical coordinate. Distinct UAVs do not simultaneously share the same trajectory, and the distance between these coordinates is longer than the trajectory radius to avoid collisions.. Once UAVs start flying, they remain powered until no more requests are submitted, or until the battery has been fully exhausted.

Users initially send requests to a base station (BS), and the BS sends the workload to a nearby UAV. To guarantee that the content will be transferred quickly, a minimum data rate for this communication must be guaranteed. The quality of transmission over a wireless channel is also variable over time, due to the UAV mobility. UAVs are only suitable for processing a request if they can support the data rate requirements regardless of locations; i.e., a UAV must meet these requirements even when located at the most distant point from the BS.

\section{B. Location of fog nodes}

The problem of locating fog nodes is a special case of the classical facility location problem. A set of locations is provided as input, and the solution determines which locations will receive a facility. In fog computing, the facilities are fog nodes, and the number of processed requests is the main objective to be maximized. The formulation can involve multiple objectives. In this paper, we aim at optimizing the requests processed, reducing the number of UAVs used, and maximizing the data rate.

The fog node location problem is an optimization problem formulated as a mathematical programming model. In the formulation, linear equations model the constraints and objectives of the problem, while integer variables represent the decisions to be made. Therefore, the fog node location problem can be formulated as an integer linear programming model. The formulation considers discrete-time, with each request originated during a time interval and is associated with a BS. The input to the formulation are the number of available UAVs, the candidate locations for deployment of fog nodes, UAV battery available capacity and the energy expended during the different activities, the number of requests made and their requirements, and the minimum data rates that a UAV in each location should guarantee for each request. The output is the location for each $\mathrm{UAV}$, the time intervals during which the UAV will be active, and the requests processed by each fog node.

\section{Spatio-Temporal UAV Fog Node Location}

Obtaining exact solutions for the fog node location problem by considering areas occupied by real deployments is timeconsuming and often impossible, especially due to main memory limitations, this means alternative solutions are called for design a proper aerial fog computing infrastructure. Any solution for the fog node location problem must address two main issues. First, UAVs must be active when the requests are made, and second, a solution must consider the limitations of fixedwing UAVs. This subsection presents the STUFog algorithm used to tackle these issues and solve a location problem with numerous requests and UAVs.

The proposed algorithm makes decisions to maximize the requests served. It accounts for the number of requests that a UAV can process at every available location during each time interval. This information allows the identification of where 


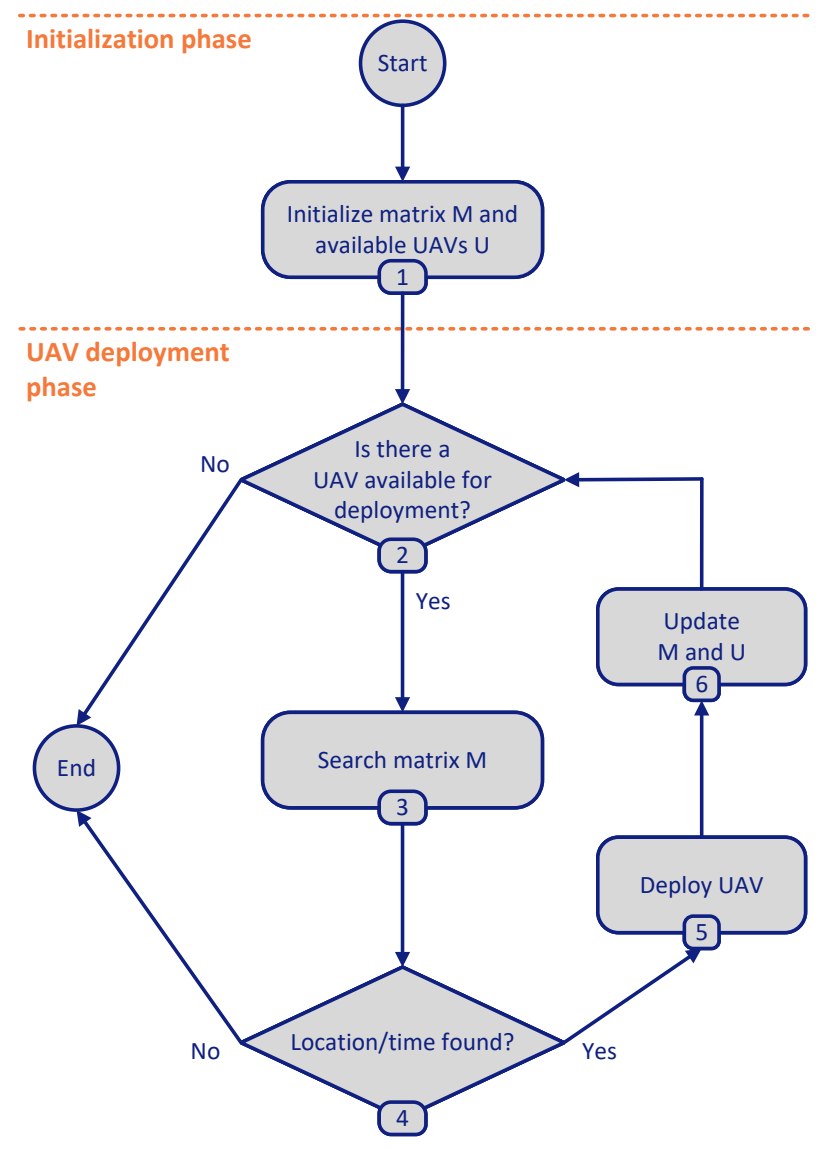

Fig. 1. Flowchart of the STUFog algorithm.

and when requests are made, positioning UAVs so that they can process most of the requests made. Moreover, the algorithm considers the physical limitations of fixed-wing UAVs, deploying them in sequential time intervals, and accounting for their battery autonomy. It also considers circular trajectories for fixed-wing UAVs and the quality of transmission of the wireless links. The algorithm avoids overdimensioning in the number of UAVs needed by not deploying a new UAVs if it will not lead to new requests being processed. To maximize the data rate, if a UAV cannot process all requests made in a single period, the algorithm favors those close to the users, thus increasing the average data rate and leaving the remaining requests for other UAVs.

The algorithm uses a matrix $M$ with spatio-temporal data related to requests. Time is discretized, and each column of $M$ represents a time interval $t$; each row represents a candidate location $l$. Each entry in $M$ contains the requests that a UAV at $l$ during time $t$ can process. The algorithm assigns sequential columns of $M$ to each $\mathrm{UAV}$, and the maximum number of columns represents the maximum time a UAV can remain operational without recharging. Furthermore, requests are included in each entry of $M$ only if a fixed-wing UAV flying at the correspondent location can assure the minimum required data rate for the request. This data structure helps to identify locations with large demands quickly, obtaining solutions to large fog infrastructures in an acceptable time frame.

Figure 1 shows the flowchart of the STUFog algorithm with the numbered steps 1-6. There are two phases: initialization and UAV deployment. The initialization (Step 1) sets the values of $U$, the number of UAVs available for deployment, and $M$. The remaining steps (2-6) constitute the UAV deployment phase, in which UAVs are deployed to locations with large demands. In Step 3, proposed solution evaluates the entries of the matrix not assigned to a UAV. These entries represent the initial time of operation for a new UAV and the candidate location for deployment. Then, the algorithm evaluates how many requests will be processed if it assigns a UAV to that location. The algorithm chooses the element of $M$ that maximizes the number of requests processed before deploying a new UAV. If the UAV cannot process all requests made during a given time interval due to limited processing capacity, requests are selected in decreasing order of data rate. The remaining requests are left for UAVs placed at different locations in future algorithm iterations.

Step 4 minimizes the number of UAVs employed: the algorithm stops deploying UAVs if all requests have been served or the current UAV cannot cope with the data rate constraints of the remaining requests. If the deployment of a new UAV is beneficial, then Step 5 deploys the UAV, and Step 6 updates $U$ and $M$, with $U$ decreased by one, and requests processed by the current UAV removed from the $M$ elements. The elements of $M$ that represent the location and time during which the 
TABLE II

PARAMETERS ADOPTED IN THE EVALUATION

\begin{tabular}{ll}
\hline \multicolumn{2}{c}{ Fixed-wing } \\
\hline Energy consumption (circular) & Model in [8] \\
Radius & 100,200, and $300 \mathrm{~m}$ \\
Speed & $30 \mathrm{~m} / \mathrm{s}$ \\
\hline \multicolumn{2}{c}{ Rotary-wing } \\
\hline Energy consumption (hovering) & Model in [9] \\
\hline \multicolumn{2}{c}{ Wireless channel } \\
\hline Channel model & Path Loss in 3GPP TR 36.777 [12] \\
Line-of-Sight probability & LoS in 3GPP TR 36.777 [12] \\
BS type & Urban macro (25 m) \\
Transmission power & $23 \mathrm{dBm}[12]$ \\
Noise & $-60 \mathrm{dBm}[7]$ \\
Bandwidth & $10 \mathrm{mHz}[12]$ \\
Required data rate & $50 \mathrm{Mbps}$ \\
\hline & Other \\
\hline parameters \\
\hline UAV altitude & $100 \mathrm{~m}$ \\
UAV battery & $34200 \mathrm{mAh} / 22.8 \mathrm{~V}$ \\
Workload & Milan data set [13] \\
BS positions & $100 \mathrm{positions} \mathrm{from} \mathrm{OpenCellId} \mathrm{[14]}$ \\
Number of locations & 100 \\
Request processing requirement & 10 processing units \\
$Z$ & 0.0005 \\
Total area & $552.25 \mathrm{~km}{ }^{2}$ \\
\hline
\end{tabular}

UAV is active are then set as used. These operations guarantee that single requests are processed only once, and two UAVs do not perform the same trajectory, thus avoiding collisions. The algorithm continues until all available UAVs have been deployed (Step 2) or the inclusion of a new UAV is ineffective (Step 4).

\section{Evaluation}

To evaluate the performance of fixed-wing UAVs as fog nodes, simulations were carried out using requests based on actual data. This section describes the simulation setup and then discusses the numerical results.

\section{A. Simulation setting}

The STUFog algorithm was coded in Python to simulate diverse UAV deployments. The present subsection describes the settings used for these simulations. Table II summarizes the values of the parameters employed. The energy consumption model is the one derived in [8]. For the sake of comparison, deployments with rotary-wing UAVs hovering continuously in the same position were also simulated. Their energy consumption model is that in [9]. Both types of UAVs have the same battery [11] and fly at $100 \mathrm{~m} \mathrm{[8],} \mathrm{[9].}$

The wireless channel is modeled using the path loss and line-of-sight probabilities given by the 3GPP model [12]. The path loss is calculated using the physical distance between UAVs and BS, and line-of-sight. The LoS probability model is also a function of the physical distance and it is calculated for all pairs of BS and locations for UAVs. The values of transmission power, noise, and bandwidth were based on previous studies [7], [12]. The Doppler effect due to the UAV motion was assumed to have been perfectly compensated for. A minimum requirement for the data rate was set to allow low-latency transmissions between BSs and UAVs. If this requirement is not met, the UAV cannot process the requests from that BS. If no UAV can serve the requests of that $\mathrm{BS}$, the requests are blocked.

The number of requests received by each base station during every time interval is obtained from the public data set provided by Telecom Italia [13], a data set containing Call Detail Records (CDR) of mobile users collected in November and December, 2013, in the metropolitan region of Milan, Italy. These data set contain the data for a 100x100 grid, where each cell in the grid has its CDR information recorded for intervals of 10 minutes. The CDR data on Internet accesses was used to obtain the number of requests. The BS locations were obtained from the OpenCellId project [14], a public database with BS data collected by mobile users. The coordinates of the BSs that were active during the period of data collection of the Milan data set [13] were obtained. The CDR data of each cell was mapped to the closest BS, as in [1]. If multiple BSs were inside a cell, their requests were equally balanced among them. The data in the data set [13] were anonymized and do not show the total number of accesses. Therefore, the integer number of requests $R$ made to each BS was obtained by using the constant $Z$. $R$ is calculated by multiplying $Z$ by the number of Internet accesses obtained from the data set.

A third database, the Shuttle Radar Topography Mission [15], was used to obtain the altitude of the BSs, thus allowing the calculation of the distance between UAVs and BSs. The locations for deployment of UAVs are based on a map of Milan and were equally distant from each other. For the fixed-wing UAVs, these locations were the center of the trajectory; for rotary-wing 


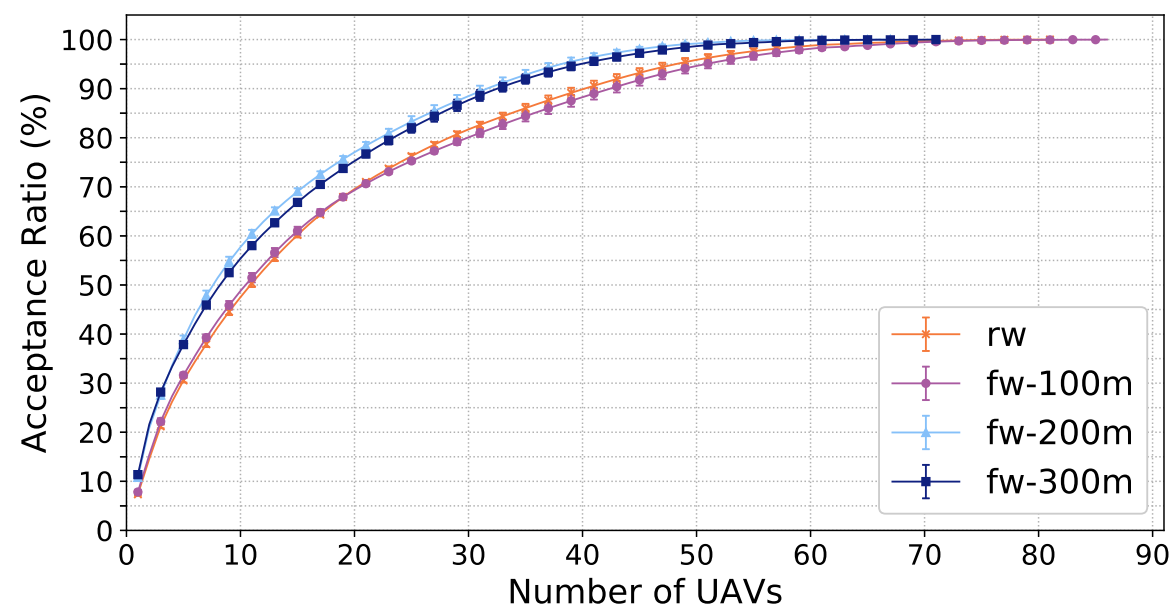

Fig. 2. Acceptance ratio produced by battery-powered UAVs.

UAVs, they were the point where the UAV hovered. The server capacity was 100 processing units per time interval, and the duration of each interval was 10 minutes, resulting in 144 intervals for every 24 hours.

\section{B. Battery power evaluation}

This subsection presents the numerical results obtained in the simulations with battery-powered UAVs. The metrics are the acceptance ratio of requests and the average data rate. The former is the ratio of the number of requests processed by fog nodes to the total number of requests. The latter is the average data rate between BS and UAV considering all processed requests and the adopted channel model. Both metrics are given as a function of the number of employed UAVs. The STUFog algorithm started with the budget of a single UAV. Subsequent executions were performed increasing the number of UAVs one by one until the inclusion of a new UAV did not lead to any new request being processed. This happens in two cases: either there are no more requests to be processed (100\% acceptance), or the available time intervals and locations do not make it possible for new UAVs to meet the requirements of the requests. The results obtained for the deployments of fixed-wing UAVs are identified as $f_{W}-R R R m$, where RRR is the radius of the circular trajectory in meters. The results for rotary-wing UAV deployments are identified as rw. 95\% confidence intervals are displayed in the graphics.

Figure 2 presents the acceptance ratio as a function of the number of UAVs. Fixed-wing UAVs with trajectories with larger radii are more energy-efficient and tend to improve the acceptance ratio by processing more requests at different times. Therefore, the acceptance ratio of fixed-wing UAVs with a 200-300 m radius was greater than that of the other deployments. The acceptance ratio of rotary-wing UAVs was similar to that of fixed-wing trajectory with $100 \mathrm{~m}$ radius, with both deployments consuming similar amounts of energy. However, energy consumption is not the only important factor. A deployment with a $200 \mathrm{~m}$ radius produced a higher acceptance ratio than did that with a radius of $300 \mathrm{~m}$, despite the greater energy efficiency of the latter. This apparent lack of correlation has been attributed to the quality of the wireless link. In deployments with a $300 \mathrm{~m}$ radius, fixed-wing UAVs can be too far from the ground BSs for the data rate provided on the wireless channel to be sufficient to deal with low-latency delay requirements. In this case, more UAVs closer to users are needed to achieve a $100 \%$ acceptance of requests.

Figure 3 shows the data rate as a function of the number of UAVs. All deployments produced an average data rate between 65 and $75 \mathrm{Mbps}$, consistently higher than the minimum required (50 Mbps), demonstrating that UAVs can provide network services with a high quality, thus helping to reduce latency. The rotary-wing UAV produced the best results for the data rate due to its stability which guarantees better wireless signals. For the fixed-wing UAV, the data rate calculated considered the point of the trajectory where the distance to the BS was maximal. Consequently, greater radius values led to only small reductions in data rate.

If only fixed-wing UAVs are available, the $200 \mathrm{~m}$ radius deployment provided the best trade-off between the acceptance of requests and wireless channel quality. However, choosing between fixed-wing and rotary-wing UAVs depends on the cost. Both types of UAV are available in various models, and prices vary significantly. Obtaining a $90 \%$ acceptance ratio value requires at least 33 fixed-wing UAVs or 42 rotary-wing UAVs. Therefore, if the cost of a rotary-wing UAV is at least 13\% lower than that of a fixed-wing one, deployments with rotary-wing UAVs are more advantageous, obtaining higher acceptance ratios than that employing fixed-wing UAVs for the same capital expenditure (CAPEX), yet providing a slightly higher data rate. 


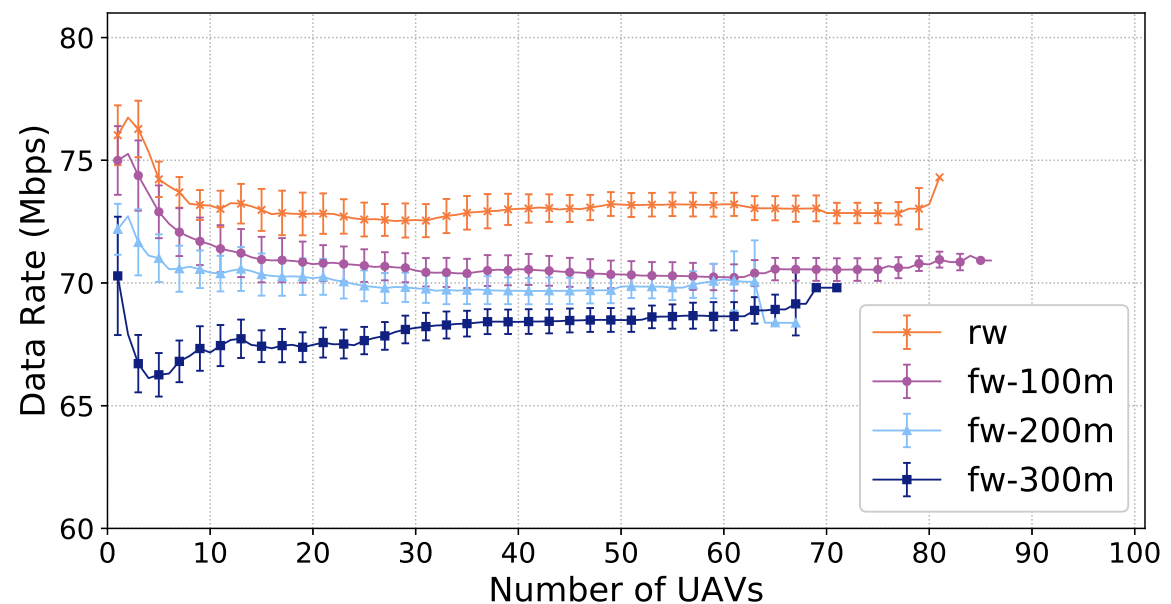

Fig. 3. Data rate of battery-powered UAVs.

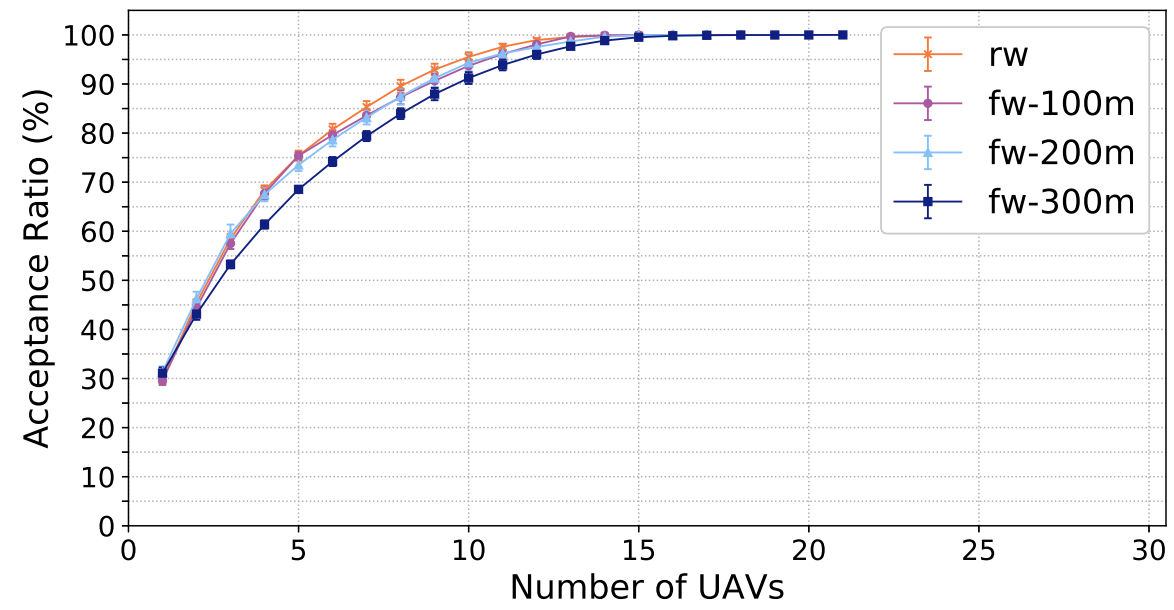

Fig. 4. Acceptance ratio produced by tethered UAVs.

\section{Tethered UAV evaluation}

Figure 4 shows the acceptance ratio for deployments of tethered UAVs. Energy is considered to be unlimited, and, consequently, the UAVs remain operational all the time. Therefore, the number of UAVs needed to achieve $100 \%$ request acceptance is significantly reduced, being at most 21 UAVs. Rotary-wing UAVs that are hovering have wireless channels with higher quality, which leads to the higher acceptance ratio for the same number of UAVs; fixed-wing UAVs with a 100 or 200-meter radius have acceptance ratio somewhat lower. Since energy consumption is not a significant concern in tethered deployments, those with rotary-wing UAVs make more sense unless they are more expensive than those of fixed-wing ones. Tethering fixedwing UAVs with a large radius is not feasible for two main reasons. First, the main reason for the adoption of fixed-wing UAVs flying with a large radius trajectory is to extend the battery lifetime, which is irrelevant for tethered deployments. Second, the length of a typical tether is in the range of 80-150m [5]. Therefore, if fixed-wing UAVs are employed, $100 \mathrm{~m}$ or smaller radii are preferred.

Tethered deployments are indeed advantageous for reducing costs, since they substantially reduce the number of required UAVs. Nonetheless, there are key limitations that can prevent their use. First, there is an extra cost involved in installing and maintaining the GSs. Fog providers may need to rent rooftops of high buildings in metropolitan areas, which considerably increases operational costs. Second, connecting UAVs to tethers requires workers who are physically present, which increases operational costs, while untethered UAVs operations can be carried out remotely. Finally, tethered UAVs are not indicated in urban environments due to the risks imposed by the tether, and are adequate to rural areas. Therefore, fog computing infrastructure with tethered UAVs is somewhat limited, but its use reduces the number of UAVs. 


\section{CONCLUSIONS}

This paper has investigated the employment of fog nodes mounted on fixed-wing UAVs. The STUFog algorithm has been proposed to locate UAVs in areas and time intervals which maximizes the number of requests processed. Simulations were performed using real traces to evaluate the effectiveness of the proposed solution. The results show that it is possible to maintain good networking conditions for the processing of requests for several users. Moreover, if the costs of a fixed-wing UAV are similar to those of a rotary-wing UAV, deployments of fixed-wing UAVs can be quite advantageous. Finally, tethered rotary-wing UAVs present advantages over fixed-wing ones, although this comes with the cost of coping with various limitations.

\section{ACKNOWLEDGMENT}

This research was funded by Google Latin American Research Awards 2020, the National Council for Scientific and Technological Development (CNPq) grant number 140464/2018-2, and the São Paulo Research Foundation (FAPESP) grant $15 / 24494-8$.

\section{REFERENCES}

[1] R. A. C. da Silva, N. L. S. da Fonseca, and R. Boutaba, "Evaluation of the employment of uavs as fog nodes," IEEE Wireless Communications, vol. 28, no. 5, pp. 20-27, 2021.

[2] F. Granelli, C. Costa, J. Zhang, R. Bassoli, and F. H. Fitzek, "Design of an on-demand agile 5G multi-access edge computing platform using aerial vehicles," IEEE Commun. Stand. Mag., vol. 4, no. 4, pp. 34-41, 2020.

[3] G. Faraci, C. Grasso, and G. Schembra, "Fog in the clouds: UAVs to provide edge computing to IoT devices," ACM Trans. Internet Technol., vol. 20, no. 3, pp. 1-26, Aug. 2020.

[4] B. Galkin, J. Kibilda, and L. A. DaSilva, "UAVs as mobile infrastructure: Addressing battery lifetime," IEEE Commun. Mag., vol. 57, no. 6, pp. 132-137, 2019.

[5] M. Kishk, A. Bader, and M.-S. Alouini, "Aerial base station deployment in 6G cellular networks using tethered drones: The mobility and endurance tradeoff," IEEE Veh. Technol. Mag., vol. 15, no. 4, pp. 103-111, 2020.

[6] M. Zhao, W. Li, L. Bao, J. Luo, Z. He, and D. Liu, "Fairness-aware task scheduling and resource allocation in UAV-enabled mobile edge computing networks," IEEE Trans. Green Commun. Netw., pp. 1-14, 2021.

[7] J. Wang, K. Liu, and J. Pan, "Online UAV-mounted edge server dispatching for mobile-to-mobile edge computing," IEEE Internet Things J., vol. 7, no. 2, pp. 1375-1386, Feb 2020.

[8] Y. Zeng and R. Zhang, "Energy-efficient UAV communication with trajectory optimization," IEEE Trans. Wireless Commun, vol. 16, no. 6, pp. 3747-3760, 2017.

[9] Y. Zeng, J. Xu, and R. Zhang, "Energy minimization for wireless communication with rotary-wing UAV," IEEE Trans. Wireless Commun, vol. 18, no. 4, pp. 2329-2345, 2019.

[10] X. Lin, V. Yajnanarayana, S. D. Muruganathan, S. Gao, H. Asplund, H.-L. Maattanen, M. Bergstrom, S. Euler, and Y.-P. E. Wang, "The sky is not the limit: LTE for unmanned aerial vehicles," IEEE Commun. Mag., vol. 56, no. 4, pp. 204-210, 2018.

[11] M. Moradi, K. Sundaresan, E. Chai, S. Rangarajan, and Z. M. Mao, "Skycore: Moving core to the edge for untethered and reliable UAV-based LTE networks," in MobiCom '18, New York, NY, USA, 2018, p. 35-49.

[12] 3GPP, "Technical Specification Group Radio Access Network; Study on Enhanced LTE Support for Aerial Vehicles," 3rd Generation Partnership Project (3GPP), Technical Report (TR) 36.777, 12 2017, version 15.0.0.

[13] G. Barlacchi, G. Barlacchi, M. De Nadai, R. Larcher, A. Casella, C. Chitic, G. Torrisi, F. Antonelli, A. Vespignani, A. Pentland, and B. Lepri, "A multi-source dataset of urban life in the city of milan and the province of trentin," Scientific Data, vol. 2, no. 1, p. 150055, Oct 2015.

[14] Opencellid. Accessed on 2021.01.12. [Online]. Available: http://www.opencellid.org

[15] T. G. Farr, P. A. Rosen, E. Caro, R. Crippen, R. Duren, S. Hensley, M. Kobrick, M. Paller, E. Rodriguez, L. Roth, D. Seal, S. Shaffer, J. Shimada, J. Umland, M. Werner, M. Oskin, D. Burbank, and D. Alsdorf, “The shuttle radar topography mission,” Reviews of Geophysics, vol. 45, no. 2, 2007.

Rodrigo A. C. da Silva is currently a Ph.D. candidate at the Institute of Computing, State University of Campinas, Brazil.

Nelson L. S. da Fonseca is a Full Professor at the Institute of Computing of the State University of Campinas, Campinas, Brazil. 